\title{
Increase in mastery levels using a computer-based tutorial/simulation in experimental psychology
}

\author{
LINDA C. PETTY \\ Hampton University, Hampton, Virginia \\ and \\ ELLEN F. ROSEN \\ The College of William and Mary, Williamsburg, Virginia
}

\begin{abstract}
A computer program was written in PCPilot to teach a unit in basic concepts in experimental design. The graphics-based program includes both tutorials and simulations. The students who used the program had significantly higher test scores than those in another class who did not; they reported that they enjoyed learning to use the program.
\end{abstract}

In the Methods I course at Hampton University, students learn basic concepts of experimental design through homework exercises on hypothesis generation, operational definitions, and design principles, after which they conduct three simple experiments, analyze the data, test their hypotheses, and write up the results in APA style. Until 1988 , this course consisted of a traditional lecture, discussion, and homework format. The exercises and experiments were conducted alone or with a partner at the student's convenience, and the student then brought the completed exercises to class, where they were discussed and critiqued in small groups; these assignments were completed during the semester's first month. The students then found partners to conduct a reaction time study. Because this was the first experiment that most of the students had ever done, considerable guidance was given in a handout that accompanied the assignment; an introduction and method section were provided. The students were expected to follow the handout to the letter in their experimental protocols. Reaction time measurement devices were made available; experimental partners signed up for the times when subjects were scheduled, and they conducted the study according to the method section. After the data were collected, each student worked independently to analyze them, using a computerized $t$ test. Finally, the student wrote the results and the discussion section, produced any graphs needed, and composed the abstract. All of the theoretical and didactic materials were covered in the readings and/or in the lecture and discussion meetings.

Students at Hampton University take this course in the first semester of their sophomore year. It is the first course

Correspondence should be addressed to Linda C. Petty, Department of Psychology, Hampton University, Hampton, VA 23368. that majors are required to take beyond introductory psychology. Although such students have had a year of biology and a semester of chemistry before entering the course, they still know nothing of the uncertainty principle or of observer bias studies, because the courses in natural science emphasize demonstrations of principles rather than hypothesis testing or confidence limits. The instructor has therefore found it necessary to help students give up their stranglehold on the idea that "seeing is believing" or that "experiencing something is absolute proof that it is a material reality"; the fastest and surest means of dealing with this dilemma has been to use illusions of all types. Class time must be used for demonstrations of such illusions and for discussion of how the structure of the body itself forever locks human beings and all other animals away from direct "observation" or "experience" of outside reality. A subsequent discussion topic then becomes, "with what would the observation be made if not the eye and brain?'" It is very difficult to get students to think along these lines, because college sophomores do not usually choose to wrestle with such questions. The instructor must therefore ensure that students master the basic aspects of experimental design and hypothesis testing, while remaining free to spend class time on the more theoretical aspects of the philosophy of science.

\section{The Program: HECBERT}

Over a 4-year period, the instructor wrote and produced a 4-h computer-based tutorial and simulation program in PCPilot, to give the students individual practice outside of class. When students initiate the program, they are welcomed to the laboratory of HECBERT, the Hampton Educational Computer-Based Experimental Research Tutor, and asked to type their names and any other identifying information that the instructor might want. They are then moved along to the program menu, which offers five choices: 
1. WHAT'S AN EXPERIMENT?

2. EXPERIMENT 1: DRUGS AND MEMORY

3. HUMAN REACTION TIME STUDIES

4. EXPERIMENT 2: REACTION TIME

5. QUIT

The first choice introduces a tutorial on the basic concepts of experimental design, including independent, dependent, situational, and subject variables, control groups, and observer bias. A study of the effects of amphetamine on digit-span memory is used to illustrate how to design a methodologically sound study. The choice of levels of the independent variable and assignment to group are explained. Through the use of attractive screens, graphics, and fill-in-the-blank questions that accept many variations on the correct answer, an interactive conversational atmosphere is maintained, and the students seem to forget that they are doing course work.

The second choice leads to a simulation of a study of the effects of amphetamine on short-term memory. Students are asked to go through the digit-span memory test procedure themselves before the simulation generates their data. They are given four numbers and asked to type them in in the same order in which they were flashed on the screen. For each number of digits, they are given 10 trials and then moved up to the next number until they miss. If they can correctly type the four numbers that they have been shown, they get 9 more trials with four random numbers and then move up to five for 10 trials and on to six digits until they miss. Their digit-span memory is defined as the highest level before they missed. After this test, they are asked to decide how many subjects they want in each of the groups that were described in Part 1. The program then generates the raw data for each subject, calculates the mean for the group, and types the mean at the bottom of the data column for the group. The mean is then defined and again typed on the screen alongside the group number. The students are asked several questions about the relationship of each group mean to the others, as well as what conclusions can be drawn from this eyeballing of the data. They are then asked to type in a description of the relationship of the means to one another in words. The module ends with a graphic tutorial on drawing histograms as an example of graphing data.

The third selection provides a brief review of the experimental literature in the area of human reaction time. As part of this discussion, there is an animation of what could be seen through the telescope of the observatory of the Astronomer General in Greenwich in 1796, which allows students to measure their own reaction times as they mark the passage of comets across crosshairs. APA style of citing references is illustrated and explained. Finally, several variables (motivation, arousal, boredom, personality, and fatigue) that are known to affect human reaction time are described in anecdotes, with examples of experimental studies. The students are asked to share such an experience in an open-ended interaction with the computerized tutor, who then reflects it back to them much as a Rogerian therapist would.

The fourth and final module is another simulation. At the outset, the students are asked to choose as an independent variable one of those discussed in the previous unit as variables that affect reaction time as their independent variable. They are asked to choose the levels of this variable and then the number of subjects in each group. The program then generates and prints out the raw data for each subject and the mean for each group. Then the students instruct the tutor in how to compare the groups using a $t$ test. The students take this data and prepare an APA style report describing the simulation and their results. They use the information in Part 3 to generate the introduction. After they complete this CAI tutorial/simulation, the students go on to conduct Studies 2 and 3 as described in the original course.

\section{Evaluation of the Program}

The instructor used the same four forms of the midterm exam in 1987 and 1988 so that a direct comparison could be made between the grades achieved by each class, in order to determine the effect of requiring interaction with this program. Fifty-seven students took the midterm exam in 1987, and 51 took the same forms in 1988. Each of the four forms had 50 multiple-choice questions drawn randomly from a pool of 250 . These forms had been found comparable during the 2 previous years when students were asked to take all four. Each form was administered in counterbalanced order, so that there would be no effect of order.

The $t$ test comparing the midterm exam grades of each of the two groups was significant $[t(106)=7.476, a=$ $.0001]$. The 1988 HECBERT group averaged about 15 points higher (65.412 vs. 50.281$)$ on a 100 -point scale than the 1987 non-HECBERT class did.

Informal interviews with the students in the class that used HECBERT revealed that they enjoyed their interaction with the computer. Several approached the instructor to tell her something that HECBERT had responded in conversation, as though she would not have known. The students accepted HECBERT as a personality and talked of visiting him in the computer laboratory. Student comments about the system revealed that the students liked the fact that they could review material at their own pace without worrying about someone thinking that they were slow. The program was designed to require the learner to be active at all times, and it counsels the students to stop and come back later if the student's answers indicate that the student is not attending. The staff of the microcomputer laboratory where the students used this program indicated that students laughed out loud several times during each session with HECBERT. They 
reported that student reactions to this experience were completely positive, even though many of the students had not been required to use a microcomputer for course work previously.

The better students were challenged to run the simulations using different independent variables under varying conditions, after they had gained an understanding of research design. The weaker students used the tutorial portion of the program more extensively to master the concepts and techniques of experimental design and then went on to run the assigned simulations. None of the students reported being lost or bored by the CAI experiences.

\section{Conclusions}

The use of HECBERT has more than achieved the modest goal of freeing class time for discussions of the philosophy of science. The computer unit has actually improved student performance on a test covering necessary basic information for designing and performing experiments in psychology. The computer-based unit has also given students who are inexperienced with computers an opportunity to have a rewarding introduction to them. The students like this method of instruction because it allows them to proceed at their own pace and to review material as needed. HECBERT is the most patient and nonjudgmental teacher that they have ever encountered. 